\title{
Finite-Time Bounded Control for Coupled Parabolic PDE-ODE Systems Subject to Boundary Disturbances
}

\author{
Manna Li iD and Weijie Mao \\ Department of Control Science and Engineering, Zhejiang University, Hangzhou 310027, China \\ Correspondence should be addressed to Weijie Mao; wjmao@zju.edu.cn
}

Received 26 August 2020; Revised 22 October 2020; Accepted 24 November 2020; Published 8 December 2020

Academic Editor: Andrzej Swierniak

Copyright ( 2020 Manna Li and Weijie Mao. This is an open access article distributed under the Creative Commons Attribution License, which permits unrestricted use, distribution, and reproduction in any medium, provided the original work is properly cited.

\begin{abstract}
In this paper, the finite-time bounded control problem for coupled parabolic PDE-ODE systems subject to time-varying boundary disturbances and to time-invariant boundary disturbances is considered. First, the concept of finite-time boundedness is extended to coupled parabolic PDE-ODE systems. A Neumann boundary feedback controller is then designed in terms of the state variables. By applying the Lyapunov-like functional method, sufficient conditions which ensure the finite-time boundedness of closed-loop systems in the presence of time-varying boundary disturbances and time-invariant boundary disturbances are provided, respectively. Finally, the issues regarding the finite-time boundedness of coupled parabolic PDE-ODE systems are converted into the feasibility of linear matrix inequalities (LMIs), and the effectiveness of the proposed results is validated with two numerical simulations.
\end{abstract}

\section{Introduction}

Problems concerning coupled systems have been interesting areas for long, exist in many control applications such as thermoelastic coupling, electromagnetic coupling, mechanical coupling, and coupled chemical reactions, and researchers have worked out fruitful results in these areas $[1,2]$. The backstepping technique, which is originally used for PDEs (partial differential equations) by Krstic and Smyshlyaev [3], has been applied to design the boundary feedback control law for first-order hyperbolic PDE-ODE (ordinary differential equation) couple systems [4]. For the boundary control problems of PDE-ODE cascades, where the PDEs are either of parabolic type or of hyperbolic type with Dirichlet interconnections, they have been extended to interconnections of Neumann type in [5]. Furthermore, the exponential stabilization of cascaded reaction-diffusion PDE-ODE systems with Neumann interconnections has been considered in [6], and the stabilization of a cascaded heat-ODE system coupling at an intermediate point, which is motivated by the thermoelastic coupling physics, has also been investigated [7]. While in practical engineering systems, external uncertain disturbances are often encountered problems which reduce the system quality. Much attention has been dedicated in the past years for the control of coupled PDE-ODE systems under the influence of external disturbances. For instance, the stabilization of the cascaded ODE-Schrodinger systems with boundary control matched disturbances has been studied in [8]. In addition, the sliding mode control (SMC) is integrated with the backstepping method to deal with the boundary feedback stabilization of a cascaded heat PDE-ODE system with the external boundary disturbance by Dirichlet/Neumann actuation [9]. However, all these achievements mentioned above mainly consider the case within an infinite time interval, and the finite-time control problem of coupled parabolic PDE-ODE systems with disturbances at the boundary control end is an inspiring area that is still wide open.

The concept of finite-time control can be dated back to the 1950's and appeared in the control literature in the West during the 1960's [10-12]. Given a bounded initial condition, a system is said to be finite-time stable if its state does not move beyond a certain domain over a specified time 
interval [13-17]. Most of the existing studies on finite-time stability mainly focused on different ordinary differential equation (ODE)-based systems, such as linear continuous systems [18, 19], nonlinear systems [20-23], discrete-time systems [24, 25], and time-varying systems [26]. It should be noted that few results related to the finite-time stability of PDE-based systems have been achieved. In [27], the definition of finite-time stability has been extended to distributed parameter systems, and sufficient conditions in terms of LMIs are given to achieve finite-time stability by statefeedback controllers. Moreover, the finite-time stabilization problem of distributed parameter systems with control acting on a Dirichlet condition (DC) boundary is discussed in [28]. With consideration of exogenous disturbances, the definition of finite-time boundedness of linear systems has been introduced by Amato et al. [29]. A system is said to be finite-time bounded if, given a bound on the initial condition and the set of disturbance inputs, its state does not exceed the prescribed limit for all admissible inputs in the set. For example, the finite-time stability and boundedness of linear time-varying systems have been considered based on the existence of Lyapunov-like functionals whose properties differ significantly from those of classical Lyapunov functions [30]. Subsequently, finite-time boundedness and stabilization of a class of networked control systems (NCSs) and switched linear systems with consideration of time delay and time-varying exogenous disturbances have been investigated in [31, 32], respectively. Motivated by the above discussions, the finite-time boundedness of coupled parabolic PDE-ODE systems subject to boundary disturbances has not been reported in the literature yet, thereby inspiring the main purpose of this research.

In this study, we consider the finite-time bounded control problem for coupled parabolic PDE-ODE systems subject to time-varying boundary disturbances and to timeinvariant boundary disturbances. Our main contribution is to design a Neumann boundary feedback control law, and sufficient conditions are provided such that the closed-loop systems in the presence of time-varying boundary disturbances and time-invariant boundary disturbances are finitetime bounded, respectively. First, the concept of finite-time boundedness is extended to coupled parabolic PDE-ODE systems. The Neumann boundary feedback controller is then derived in terms of matrix inequalities, which guarantee the finite-time boundedness of the considered systems. Finally, the proposed conditions are converted into the feasibility of linear matrix inequalities (LMIs), and the availability of this method is verified through numerical simulations.

The remainder of this paper is presented as follows. Section 2 states the problem formulations and some preliminaries. Section 3 is devoted to the design of the Neumann boundary feedback controller, and sufficient conditions for the finite-time boundedness of closed-loop parabolic PDE-ODE cascades are provided. Section 4 gives two numerical examples to illustrate the effectiveness of our results. Some concluding remarks are presented in Section 5.

Notation. Let $\mathbb{R}^{n}$ denote the $n$-dimensional real space, $\mathbb{R}_{+}$denote the set of nonnegative numbers, and $\mathbb{R}^{n \times n}$ denote the set of $n \times n$ real matrices. In addition, $L^{2}([0,1])$ stands

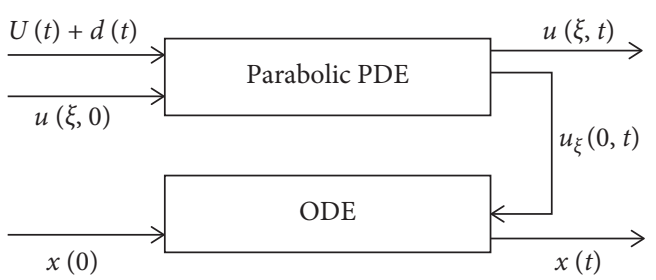

FIgURE 1: Block diagram for the coupled parabolic PDE-ODE system with Neumann interconnection.

for the Hilbert space of square integrable functions defined over $[0,1]$, and $W^{1,2}([0,1])$ denotes the Sobolev space of absolutely continuous functions with square integrable firstorder derivatives defined over $[0,1] . P>0(P<0)$ denotes the symmetric matrix $P$ is positive definite (negative definite). $\lambda_{\max }(P)\left(\lambda_{\min }(P)\right)$ represents the maximal (minimal) eigenvalue of a matrix $P$. $I_{n \times n}$ means an $n \times n$ identity matrix. The symbol $*$ is the symmetry term in a symmetric matrix.

\section{Problem Statement and Preliminaries}

In this study, we consider the coupled PDE-ODE systems with Neumann interconnection, which is of the vector form:

$$
\left\{\begin{array}{l}
x(t)=A x(t)+B u_{\xi}(0, t) \\
u_{t}(\xi, t)=D u_{\xi \xi}(\xi, t)+G u(\xi, t), \\
u(0, t)=0 \\
u_{\xi}(1, t)=U(t)+d(t) \\
\xi \in(0,1), \quad t \in[0, T]
\end{array}\right.
$$

where $x(t) \in \mathbb{R}^{n}$ is the state vector of the ODE subsystem and $u(\xi, t) \in \mathbb{R}^{n}$ is the state vector of the PDE subsystem. $U(t)$ is the control input to the entire system. $A \in \mathbb{R}^{n \times n}$ and $B \in \mathbb{R}^{n \times n}$ satisfy that the system pair $(A, B)$ is controllable. $D=\operatorname{diag}\left\{d_{i}>0\right\}$ is the diagonal matrix for all $i=1,2, \ldots n$, and $G \in \mathbb{R}^{n \times n}$ is a real-valued square matrix. $d(t) \in \mathbb{R}^{n}$ is the external disturbance at the control end, which is supposed to be bounded. $u_{t}(\xi, t)$ is the first-order partial derivative with respect to $t$, and $u_{\xi \xi}(\xi, t)$ is the second-order partial derivative with respect to $\xi$. The initial values $x(0)=x_{0}$ and $u(\xi, 0)=u_{0}(\xi)$. The whole system is depicted in Figure 1 .

Remark 1. The coupled PDE-ODE system (1) is composed of a parabolic PDE and a linear ODE, which has rich physical applications and is used to describe a widespread family of problems in science such as thermoelastic coupling. Thermoelastic coupling is an interesting phenomenon which has been extensively applied in the community of micromechanics and microengineering [2, 7]. For instance, a simplified thermoelastic system can be modeled by a cascade of a heat PDE and a linear ODE, where the state of PDE subsystem represents the temperature of a rod and the state of ODE subsystem describes the displacement of a mechanical oscillator which can be manipulated by a thermostress related to the temperature of the rod [7].

The Neumann boundary feedback controller in this study is designed as follows: 


$$
U(t)=K_{1} x(t)+\int_{0}^{1} K_{2} u(\xi, t) \mathrm{d} \xi,
$$

where $K_{1} \in \mathbb{R}^{n \times n}$ and $K_{2} \in \mathbb{R}^{n \times n}$ are two controller gain matrices to be determined.

The aim of this study is to design the Neumann boundary feedback controller, and sufficient conditions for the finitetime boundedness of the closed-loop parabolic PDE-ODE couples with time-varying boundary disturbances and timeinvariant boundary disturbances are given in terms of matrix inequalities. To obtain the solutions of the controller gains $K_{1}$ and $K_{2}$ in equation (2), these sufficient conditions are converted into the feasibility problem of linear matrix inequalities (LMIs).

Let $1 \leq p \leq \infty$, and $L^{p}([0,1])$ denotes the Hilbert space of $p^{\text {th }}$ power integrable scalar functions $z(\xi, t)$ defined over $[0,1]$ with the norm:

$$
\begin{aligned}
& \|z(\xi, t)\|_{L^{p}}=\left(\int_{0}^{1}|z(\xi, t)|^{p} \mathrm{~d} \xi\right)^{(1 / p)}, \quad 1 \leq p<\infty, \\
& \|z(\xi, t)\|_{L^{\infty}}=\operatorname{esssup}_{\xi \in[0,1]}|z(\xi, t)|, \quad p=\infty .
\end{aligned}
$$

It should be pointed out that the definition of the $L^{p}$ norm of a scalar function $z(\xi, t)$ can be extended to the vector function $u(\xi, t)$, which has been extensively applied throughout this study.

For $p=2$, and a positive definite diagonal matrix $R$, the $L^{2}$-norm of a vector function $u(\xi, t)$ is defined as

$$
\|u(\xi, t)\|_{L^{2}, R}=\left(\int_{0}^{1} u^{T}(\xi, t) R u(\xi, t) \mathrm{d} \xi\right)^{(1 / 2)}
$$

Definition 1. Given positive constants $c_{1}, c_{2}\left(c_{1}<c_{2}\right)$, and $T$, a positive definite diagonal matrix $R$, and a class of signals $W$, if there exists a boundary feedback controller $U(t)$, the closed-loop system (1) is said to be finite-time bounded with respect to $\left(c_{1}, c_{2}, T, R, W\right)$, if

$$
\begin{aligned}
& x^{T}(0) R x(0)+\|u(\xi, 0)\|_{L^{2}, R}^{2}<c_{1}^{2} \Longrightarrow x^{T}(t) R x(t) \\
& +\|u(\xi, t)\|_{L^{2}, R}^{2}<c_{2}^{2}, \quad \forall t \in[0, T],
\end{aligned}
$$

for all $d(\cdot) \in W$.

Remark 2. Note that the concept of finite-time boundedness is induced from finite-time stability in the presence of exogenous disturbance inputs [30], which is quite different from the idea of Lyapunov asymptotic stability [33].

Lemma 1 (see [34]). For a vector function $u(\xi) \in W^{1,2}\left([0,1] ; \mathbb{R}^{n}\right)$, if $u(0)=0$ or $u(1)=0$, for a symmetric positive definite matrix $P \in \mathbb{R}^{n \times n}$, the integral inequality holds:

$$
\int_{0}^{1} u^{T}(\xi) P u(\xi) \mathrm{d} x \leq \frac{4}{\pi^{2}} \int_{0}^{1} u_{\xi}^{T}(\xi) P u_{\xi}(\xi) \mathrm{d} \xi .
$$

\section{Main Results}

3.1. Finite-Time Boundedness of the Coupled Parabolic PDEODE Systems Subject to Time-Varying Boundary Disturbances

Theorem 1. Consider the following class of signals:

$$
W:=\left\{d(\cdot) \mid d(\cdot) \in L^{2}([0, T]), \int_{0}^{T} d^{T}(t) d(t) \mathrm{d} t \leq w\right\},
$$

where $L^{2}([0, T])$ is the set of square integrable vector valued functions in $[0, T]$ and $w$ is a positive scalar. Then, under the boundary feedback controller $U(t)$, system (1) is finite-timebounded with respect to $\left(c_{1}, c_{2}, T, R, W\right)$, if, letting $\widetilde{P}=R^{-(1 / 2)} P R^{-(1 / 2)}$, there exists a positive definite symmetric matrix $P$, and the controller gain matrices are $K_{1}$ and $K_{2}$ and positive scalars $\alpha, \beta$, and $\gamma$ such that

$$
\left[\begin{array}{ccc}
\Xi_{1} & P D K_{1} & -P D K_{1} \\
* & \Xi_{2} & -K_{2}^{T} D^{T} P \\
* & * & -\frac{\pi^{2}}{4}\left(D^{T} P+P D\right)+\frac{1}{\gamma}(D P)^{T} D P
\end{array}\right]<0,
$$

where

$$
\begin{aligned}
& \Xi_{1}=P\left(A+B K_{1}\right)+\left(A+B K_{1}\right)^{T} P+\frac{1}{\beta} P B B^{T} P-\alpha P, \\
& \Xi_{2}=P\left(D K_{2}+G\right)+\left(D K_{2}+G\right)^{T} P-\alpha P .
\end{aligned}
$$

Proof. We choose a Lyapunov functional as

$$
V(t)=V_{1}(t)+V_{2}(t)
$$

where

$$
\begin{aligned}
& V_{1}(t)=x^{T}(t) P x(t), \\
& V_{2}(t)=\int_{0}^{1} u^{T}(\xi, t) P u(\xi, t) \mathrm{d} \xi .
\end{aligned}
$$

The time derivative of $V_{1}(t)$ along the trajectory of system (1) is given as

$$
\begin{aligned}
\dot{V}_{1}(t)= & x^{T}(t) P \dot{x}(t)+\dot{x}^{T}(t) P x(t) \\
= & x^{T}(t) P A x(t)+x^{T}(t) P B u_{\xi}(0, t)+x^{T}(t) A^{T} P x(t) \\
& +u_{\xi}^{T}(0, t) B^{T} P x(t) .
\end{aligned}
$$

Based on (1) and (2), we obtain that

$$
u_{\xi}(0, t)=K_{1} x(t)+d(t)
$$


Then, expression (14) can be written as

$$
\begin{aligned}
\dot{V}_{1}(t)= & x^{T}(t) P A x(t)+x^{T}(t) P B K_{1} x(t)+x^{T}(t) P B d(t)+x^{T}(t) A^{T} P x(t) \\
& +x^{T}(t) K_{1}^{T} B^{T} P x(t)+d(t)^{T} B^{T} P x(t) \\
= & x^{T}(t)\left[P\left(A+B K_{1}\right)+\left(A+B K_{1}\right)^{T} P\right] x(t)+x^{T}(t) P B d(t)+d^{T}(t) B^{T} P x(t) \\
= & x^{T}(t)\left[P\left(A+B K_{1}\right)+\left(A+B K_{1}\right)^{T} P+\frac{1}{\beta} P B B^{T} P\right] x(t)+\beta d^{T}(t) d(t) \\
& -\left(\sqrt{\beta} d(t)-\frac{1}{\sqrt{\beta}} B^{T} P x(t)\right)^{T}\left(\sqrt{\beta} d(t)-\frac{1}{\sqrt{\beta}} B^{T} P x(t)\right) \\
\leq & x^{T}(t)\left[P\left(A+B K_{1}\right)+\left(A+B K_{1}\right)^{T} P+\frac{1}{\beta} P B B^{T} P\right] x(t)+\beta d^{T}(t) d(t) .
\end{aligned}
$$

Subsequently, the time derivative of $V_{2}(t)$ along the trajectory of system (1) is presented as

$$
\begin{aligned}
\dot{V}_{2}(t) & =\int_{0}^{1} u^{T}(\xi, t) P u_{t}(\xi, t) \mathrm{d} \xi+\int_{0}^{1} u_{t}^{T}(\xi, t) P u(\xi, t) \mathrm{d} \xi \\
& =\int_{0}^{1} u^{T}(\xi, t) P D u_{\xi \xi}(\xi, t) \mathrm{d} \xi+\int_{0}^{1} u_{\xi \xi}^{T}(\xi, t) D^{T} P u(\xi, t) \mathrm{d} \xi+\int_{0}^{1} u^{T}(\xi, t)\left(P G+G^{T} P\right) u(\xi, t) \mathrm{d} \xi
\end{aligned}
$$

Applying the integration by parts, and the fact that $u(0, t)=0$, expression (17) can be written as

$$
\begin{aligned}
\dot{V}_{2}(t)= & \int_{0}^{1} u^{T}(1, t) P D K_{1} x(t) \mathrm{d} \xi+\int_{0}^{1} u^{T}(1, t) P D K_{2} u(\xi, t) \mathrm{d} \xi+\int_{0}^{1} x^{T}(t) K_{1}^{T} D^{T} P u(1, t) \mathrm{d} \xi \\
& +\int_{0}^{1} u(\xi, t)^{T} K_{2}^{T} D^{T} P u(1, t) \mathrm{d} \xi+\int_{0}^{1} u^{T}(1, t) P D d(t) \mathrm{d} \xi+\int_{0}^{1} d^{T}(t) D^{T} P u(1, t) \mathrm{d} \xi \\
& +\int_{0}^{1} u^{T}(\xi, t)\left(P G+G^{T} P\right) u(\xi, t) \mathrm{d} \xi-\int_{0}^{1} u_{\xi}^{T}(\xi, t)\left(D^{T} P+P D\right) u_{\xi}(\xi, t) \mathrm{d} \xi .
\end{aligned}
$$

Let $\bar{u}(\xi, t)=u(\xi, t)-u(1, t)$, it can be easily obtained that

$$
\bar{u}_{\xi}(\xi, t)=u_{\xi}(\xi, t)
$$




$$
\begin{aligned}
\dot{V}_{2}(t)= & \int_{0}^{1} u^{T}(\xi, t)\left[P\left(D K_{2}+G\right)+\left(D K_{2}+G\right)^{T} P\right] u(\xi, t) \mathrm{d} \xi-\int_{0}^{1} \bar{u}^{T}(\xi, t) P D K_{1} x(t) \mathrm{d} \xi \\
& -\int_{0}^{1} x^{T}(t) K_{1}^{T} D^{T} P \bar{u}(\xi, t) \mathrm{d} \xi+\int_{0}^{1} u^{T}(\xi, t) P D K_{1} x(t) \mathrm{d} \xi \\
& +\int_{0}^{1} x^{T}(t) K_{1}^{T} D^{T} P u(\xi, t) \mathrm{d} \xi-\int_{0}^{1} \bar{u}^{T}(\xi, t) P D d(t) \mathrm{d} \xi \\
& -\int_{0}^{1} d^{T}(t) D^{T} P \bar{u}(\xi, t) \mathrm{d} \xi-\int_{0}^{1} \bar{u}^{T}(\xi, t) P D K_{2} u(\xi, t) \mathrm{d} \xi \\
& -\int_{0}^{1} u(\xi, t)^{T} K_{2}^{T} D^{T} P \bar{u}(\xi, t) \mathrm{d} \xi-\int_{0}^{1} \bar{u}_{\xi}^{T}(\xi, t)\left(D^{T} P+P D\right) \bar{u}_{\xi}(\xi, t) \mathrm{d} \xi
\end{aligned}
$$

Assuming that $D^{T} P+P D>0$, and from Lemma 1, equation (20) can be represented as

$$
\begin{aligned}
\dot{V}_{2}(t) \leq & \int_{0}^{1} u^{T}(\xi, t)\left[P\left(D K_{2}+G\right)+\left(D K_{2}+G\right)^{T} P\right] u(\xi, t) \mathrm{d} \xi \\
& -\int_{0}^{1} \bar{u}^{T}(\xi, t) P D K_{1} x(t) \mathrm{d} \xi-\int_{0}^{1} x^{T}(t) K_{1}^{T} D^{T} P \bar{u}(\xi, t) \mathrm{d} \xi \\
& +\int_{0}^{1} u^{T}(\xi, t) P D K_{1} x(t) \mathrm{d} \xi+\int_{0}^{1} x^{T}(t) K_{1}^{T} D^{T} P u(\xi, t) \mathrm{d} \xi \\
& -\int_{0}^{1} \bar{u}^{T}(\xi, t) P D d(t) \mathrm{d} \xi-\int_{0}^{1} d^{T}(t) D^{T} P \bar{u}(\xi, t) \mathrm{d} \xi \\
& -\int_{0}^{1} \bar{u}^{T}(\xi, t) P D K_{2} u(\xi, t) \mathrm{d} \xi-\int_{0}^{1} u(\xi, t)^{T} K_{2}^{T} D^{T} P \bar{u}(\xi, t) \mathrm{d} \xi \\
& -\frac{\pi^{2}}{4} \int_{0}^{1} \bar{u}^{T}(\xi, t)\left(D^{T} P+P D\right) \bar{u}(\xi, t) \mathrm{d} \xi .
\end{aligned}
$$

Then,

$$
\begin{aligned}
\dot{V}_{2}(t) \leq & \int_{0}^{1} u^{T}(\xi, t)\left[P\left(D K_{2}+G\right)+\left(D K_{2}+G\right)^{T} P\right] u(\xi, t) \mathrm{d} \xi \\
& -\int_{0}^{1} \bar{u}^{T}(\xi, t) P D K_{1} x(t) \mathrm{d} \xi-\int_{0}^{1} x^{T}(t) K_{1}^{T} D^{T} P \bar{u}(\xi, t) \mathrm{d} \xi \\
& +\int_{0}^{1} u^{T}(\xi, t) P D K_{1} x(t) \mathrm{d} \xi+\int_{0}^{1} x^{T}(t) K_{1}^{T} D^{T} P u(\xi, t) \mathrm{d} \xi \\
& -\int_{0}^{1} \bar{u}^{T}(\xi, t) P D K_{2} u(\xi, t) \mathrm{d} \xi-\int_{0}^{1} u(\xi, t)^{T} K_{2}^{T} D^{T} P \bar{u}(\xi, t) \mathrm{d} \xi \\
& +\int_{0}^{1} \bar{u}^{T}(\xi, t)\left[-\frac{\pi^{2}}{4}\left(D^{T} P+P D\right)+\frac{1}{\gamma}(D P)^{T} D P\right] \bar{u}(\xi, t) \mathrm{d} \xi \\
& -\int_{0}^{1}\left(\sqrt{\gamma} d(t)-\frac{1}{\sqrt{\gamma}} D P \bar{u}(\xi, t)\right)^{T}\left(\sqrt{\gamma} d(t)-\frac{1}{\sqrt{\gamma}} D P \bar{u}(\xi, t)\right) \mathrm{d} \xi+\gamma d^{T}(t) d(t) .
\end{aligned}
$$


Combining (16) and (22), we have

$$
\begin{aligned}
& \dot{V}(t)-\alpha V(t) \\
& \leq \int_{0}^{1}\left[\begin{array}{c}
X^{T}(t) \\
u^{T}(\xi, t) \\
\bar{u}^{T}(\xi, t)
\end{array}\right]\left[\begin{array}{ccc}
\Xi_{1} & P D K_{1} & -P D K_{1} \\
* & \Xi_{2} & -K_{2}^{T} D^{T} P \\
* & * & -\frac{\pi^{2}}{4}\left(D^{T} P+P D\right)+\frac{1}{\gamma}(D P)^{T} D P
\end{array}\right]\left[\begin{array}{c}
X^{T}(t) \\
u^{T}(\xi, t) \\
\bar{u}^{T}(\xi, t)
\end{array}\right] d \xi \\
& +(\beta+\gamma) d^{T}(t) d(t),
\end{aligned}
$$

where

$$
\begin{aligned}
& \Xi_{1}=\left[P\left(A+B K_{1}\right)+\left(A+B K_{1}\right)^{T} P+\frac{1}{\beta} P B B^{T} P-\alpha P\right], \\
& \Xi_{2}=P\left(D K_{2}+G\right)+\left(D K_{2}+G\right)^{T} P-\alpha P .
\end{aligned}
$$

In view of conditions (8) and (9), we found that

$$
\dot{V}(t)-\alpha V(t)<(\beta+\gamma) d^{T}(t) d(t) .
$$

Multiplying both the left side and right side of inequality (25) by a strictly positive function $e^{-\alpha t}$, we obtain

$$
\begin{aligned}
e^{-\alpha t}[\dot{V}(t)-\alpha V(t)] & =\frac{\mathrm{d}}{\mathrm{d} t}\left[e^{-\alpha t} V(t)\right] \\
& <e^{-\alpha t}(\beta+\gamma) d^{T}(t) d(t) \\
& \leq(\beta+\gamma) d^{T}(t) d(t) .
\end{aligned}
$$

Integrating both sides of the inequality (26) from 0 to $t$, it gives

$$
\begin{aligned}
e^{-\alpha t} V(t)-V(0) & <\int_{0}^{T}(\beta+\gamma) d^{T}(t) d(t) \mathrm{d} t \\
& \leq(\beta+\gamma) w .
\end{aligned}
$$

Then, inequality (27) is represented as

$$
V(t)<e^{\alpha t}[V(0)+(\beta+\gamma) w]
$$

Making $\widetilde{P}=R^{-(1 / 2)} P R^{-(1 / 2)}$, we have

$$
\begin{aligned}
& \lambda_{\min }(\widetilde{P})\left[x^{T}(t) R x(t)+\int_{0}^{1} u^{T}(\xi, t) R u(\xi, t) \mathrm{d} \xi\right] \\
& \leq x^{T}(t) P x(t)+\int_{0}^{1} u^{T}(\xi, t) P u(\xi, t) \mathrm{d} \xi<e^{\alpha t}[V(0)+(\beta+\gamma) w] \\
& x^{T}(0) P x(0)+\int_{0}^{1} u^{T}(\xi, 0) P u(\xi, 0) \mathrm{d} \xi \\
& \leq \lambda_{\max }(\widetilde{P})\left(x^{T}(0) R x(0)+\|u(\xi, 0)\|_{L_{2}, R}^{2}\right) .
\end{aligned}
$$

It is known that

$$
\begin{aligned}
& x^{T}(t) R x(t)+\| u\left(\xi, t \|_{L_{2}, R}^{2}\right. \\
& <\frac{e^{\alpha t}\left[\lambda_{\max }(\widetilde{P})\left(x^{T}(0) R x(0)+\|u(\xi, 0)\|_{L_{2}, R}^{2}\right)+(\beta+\gamma) w\right]}{\lambda_{\min }(\widetilde{P})} .
\end{aligned}
$$

From condition (10), we get the conclusion

$$
x^{T}(t) R x(t)+\|u(\xi, t)\|_{L_{2}, R}^{2}<c_{2}^{2} .
$$

The proof is completed.

Corollary 1. The finite-time boundedness problem from Theorem 1 is solvable if there exists a positive definite symmetric matrix $S$, the matrices $L_{1}$ and $L_{2}$, and positive scalars $\beta$, $\gamma$, and $\lambda_{1}$. By fixing a nonnegative scalar $\alpha$, the following LMIs hold:

$$
\begin{aligned}
& {\left[\begin{array}{ccccc}
\Phi_{1} & D L_{1} & -D L_{1} & B & 0 \\
* & \Phi_{2} & -L_{2}^{T} D^{T} & 0 & 0 \\
* & * & -\frac{\pi^{2}}{4}\left(S D^{T}+D S\right) & 0 & D \\
* & * & * & -\beta I & 0 \\
* & * & * & * & -\gamma I
\end{array}\right]<0,} \\
& S D^{T}+D S>0, \\
& S-R^{-1}<0, \\
& {\left[\begin{array}{cc}
-S & R^{-(1 / 2)} \\
* & -\lambda_{1} I
\end{array}\right]<0,}
\end{aligned}
$$




$$
\lambda_{1} c_{1}^{2}+(\beta+\gamma) w<c_{2}^{2} e^{-\alpha T}
$$

where

$$
\begin{aligned}
& \Phi_{1}=A S+S A^{T}+B L_{1}+L_{1}^{T} B^{T}-\alpha S \\
& \Phi_{2}=G S+S G^{T}+D L_{2}+L_{2}^{T} D^{T}-\alpha S .
\end{aligned}
$$

Proof. Let $S=P^{-1}$, pre- and postmultiply (8) by $S$. Set $L_{1}=$ $K_{1} S$ and $L_{2}=K_{2} S$. Condition (32) is equivalent to condition (8) holding by Schur implement. In addition, condition (33) can be transformed to (9) with $S=P^{-1}$.

Making $\widetilde{P}=R^{-(1 / 2)} P R^{-(1 / 2)}$, and by imposing the condition

$$
I<\widetilde{P}<\lambda_{1} I
$$

where $\lambda_{1}$ is a positive scalar, we have

$$
\lambda_{1}^{-1} R^{-1}<S<R^{-1}
$$

which is equivalent to (34) and (35) hold.

In this case, the matrix $P=S^{-1}$, and the boundary feedback controller gains are $K_{1}=L_{1} S^{-1}$ and $K_{2}=L_{2} S^{-1}$.

The proof is completed.

\subsection{Finite-Time Boundedness of the Coupled Parabolic PDE-} ODE Systems Subject to Time-Invariant Boundary Disturbances. In this section, we consider the special case relative to the previous criterion, namely, the finite-time bounded control of the coupled parabolic PDE-ODE systems subject to time-invariant boundary disturbances. The essential difference between the previous criterion and the present one is that the conservatism of the derived criterion can be reduced in the presence of unknown time-invariant boundary disturbances other than time-varying boundary disturbances.

Theorem 2. Consider the following class of signals

$$
W:=\left\{d^{T} d \leq w, \quad w>0\right\} .
$$

Then, under the boundary feedback controller $U(t)$, system (1) is finite-time-bounded with respect to $\left(c_{1}, c_{2}, T, R, W\right)$, if, letting $\widetilde{P}=R^{-(1 / 2)} P R^{-(1 / 2)}$, there exist two positive definite symmetric matrices $P$ and $Q$, the controller gain matrices $K_{1}$ and $K_{2}$, and a positive scalar $\alpha$ such that

$$
\begin{aligned}
& {\left[\begin{array}{cccc}
\Xi_{1} & P D K_{1} & -P D K_{1} & P B \\
* & \Xi_{2} & -K_{2}^{T} D^{T} P & 0 \\
* & * & -\frac{\pi^{2}}{4}\left(D^{T} P+P D\right) & -P D \\
* & * & * & -\alpha Q
\end{array}\right]<0,} \\
& D^{T} P+P D>0
\end{aligned}
$$

$$
\frac{\lambda_{\max }(\widetilde{P}) c_{1}^{2}+\lambda_{\max }(Q) w}{\lambda_{\min }(\widetilde{P})}<c_{2}^{2} e^{-\alpha T},
$$

where

$$
\begin{aligned}
& \Xi_{1}=P\left(A+B K_{1}\right)+\left(A+B K_{1}\right)^{T} P-\alpha P, \\
& \Xi_{2}=P\left(D K_{2}+G\right)+\left(D K_{2}+G\right)^{T} P-\alpha P .
\end{aligned}
$$

Proof. We choose the Lyapunov functional as

$$
V(t)=V_{1}+V_{2}+V_{3}
$$

where

$$
\begin{aligned}
V_{1} & =x^{T}(t) P x(t), \\
V_{2} & =\int_{0}^{1} u^{T}(\xi, t) P u(\xi, t) \mathrm{d} \xi, \\
V_{3} & =d^{T} Q d .
\end{aligned}
$$

The time derivative of $V_{1}, V_{2}$, and $V_{3}$ along the trajectory of system (1) is given as follows:

$$
\begin{aligned}
\dot{V}_{1}(t)= & x^{T}(t) P \dot{x}(t)+\dot{x}^{T}(t) P x(t) \\
= & x^{T}(t) P A x(t)+x^{T}(t) P B u_{\xi}(0, t) \\
& +x^{T}(t) A^{T} P x(t)+u_{\xi}^{T}(0, t) B^{T} P x(t) .
\end{aligned}
$$

Based on (1) and (2), we obtain that

$$
u_{\xi}(0, t)=K_{1} x(t)+d
$$

Expression (47) can be represented as

$$
\begin{aligned}
\dot{V}_{1}= & x^{T}(t) P A x(t)+x^{T}(t) P B K_{1} x(t) \\
& +x^{T}(t) P B d+x^{T}(t) A^{T} P x(t) \\
& +x^{T}(t) K_{1}^{T} B^{T} P x(t)+d^{T} B^{T} P x(t) \\
= & x^{T}(t)\left[P\left(A+B K_{1}\right)+\left(A+B K_{1}\right)^{T} P\right] x(t) \\
& +x^{T}(t) P B d+d^{T} B^{T} P x(t) .
\end{aligned}
$$

Then,

$$
\begin{aligned}
\dot{V}_{2}(t)= & \int_{0}^{1} u^{T}(\xi, t) P u_{t}(\xi, t) \mathrm{d} \xi+\int_{0}^{1} u_{t}^{T}(\xi, t) P u(\xi, t) \mathrm{d} \xi \\
= & \int_{0}^{1} u^{T}(\xi, t) P D u_{\xi \xi}(\xi, t) \mathrm{d} \xi \\
& +\int_{0}^{1} u_{\xi \xi}^{T}(\xi, t) D^{T} P u(\xi, t) \mathrm{d} \xi \\
& +\int_{0}^{1} u^{T}(\xi, t)\left(P G+G^{T} P\right) u(\xi, t) \mathrm{d} \xi .
\end{aligned}
$$

Note that $u(0, t)=0$, and by using integration by parts, expression (50) can be written as 


$$
\begin{aligned}
\dot{V}_{2}(t)= & \int_{0}^{1} u^{T}(1, t) P D K_{1} x(t) \mathrm{d} \xi+\int_{0}^{1} u^{T}(1, t) P D K_{2} u(\xi, t) \mathrm{d} \xi \\
& +\int_{0}^{1} x^{T}(t) K_{1}^{T} D^{T} P u(1, t) \mathrm{d} \xi \\
& +\int_{0}^{1} u(\xi, t)^{T} K_{2}^{T} D^{T} P u(1, t) \mathrm{d} \xi+\int_{0}^{1} u^{T}(1, t) P D d \mathrm{~d} \xi+\int_{0}^{1} d^{T} D^{T} P u(1, t) \mathrm{d} \xi \\
& +\int_{0}^{1} u^{T}(\xi, t)\left(P G+G^{T} P\right) u(\xi, t) \mathrm{d} \xi-\int_{0}^{1} u_{\xi}^{T}(\xi, t)\left(D^{T} P+P D\right) u_{\xi}(\xi, t) \mathrm{d} \xi .
\end{aligned}
$$

Let $\bar{u}(\xi, t)=u(\xi, t)-u(1, t)$, and from equation (19), we found that

$$
\begin{aligned}
\dot{V}_{2}(t)= & \int_{0}^{1} u^{T}(\xi, t)\left[P\left(D K_{2}+G\right)+\left(D K_{2}+G\right)^{T} P\right] u(\xi, t) \mathrm{d} \xi \\
& -\int_{0}^{1} \bar{u}^{T}(\xi, t) P D K_{1} x(t) \mathrm{d} \xi-\int_{0}^{1} x^{T}(t) K_{1}^{T} D^{T} P \bar{u}(\xi, t) \mathrm{d} \xi \\
& +\int_{0}^{1} u^{T}(\xi, t) P D K_{1} x(t) \mathrm{d} \xi+\int_{0}^{1} x^{T}(t) K_{1}^{T} D^{T} P u(\xi, t) \mathrm{d} \xi \\
& -\int_{0}^{1} \bar{u}^{T}(\xi, t) P D d \mathrm{~d} \xi-\int_{0}^{1} d^{T} D^{T} P \bar{u}(\xi, t) \mathrm{d} \xi \\
& -\int_{0}^{1} \bar{u}^{T}(\xi, t) P D K_{2} u(\xi, t) \mathrm{d} \xi-\int_{0}^{1} u(\xi, t)^{T} K_{2}^{T} D^{T} P \bar{u}(\xi, t) \mathrm{d} \xi \\
& -\int_{0}^{1} \bar{u}_{\xi}^{T}(\xi, t)\left(D^{T} P+P D\right) \bar{u}_{\xi}(\xi, t) \mathrm{d} \xi .
\end{aligned}
$$

Assuming that $D^{T} P+P D>0$, the following inequality is obtained by Lemma 1 :

$$
\begin{aligned}
\dot{V}_{2}(t) \leq & \int_{0}^{1} u^{T}(\xi, t)\left[P\left(D K_{2}+G\right)+\left(D K_{2}+G\right)^{T} P\right] u(\xi, t) \mathrm{d} \xi \\
& -\int_{0}^{1} \bar{u}^{T}(\xi, t) P D K_{1} x(t) \mathrm{d} \xi-\int_{0}^{1} x^{T}(t) K_{1}^{T} D^{T} P \bar{u}(\xi, t) \mathrm{d} \xi \\
& +\int_{0}^{1} u^{T}(\xi, t) P D K_{1} x(t) \mathrm{d} \xi+\int_{0}^{1} x^{T}(t) K_{1}^{T} D^{T} P u(\xi, t) \mathrm{d} \xi \\
& -\int_{0}^{1} \bar{u}^{T}(\xi, t) P D d \mathrm{~d} \xi-\int_{0}^{1} d^{T} D^{T} P \bar{u}(\xi, t) \mathrm{d} \xi \\
& -\int_{0}^{1} \bar{u}^{T}(\xi, t) P D K_{2} u(\xi, t) \mathrm{d} \xi-\int_{0}^{1} u(\xi, t)^{T} K_{2}^{T} D^{T} P \bar{u}(\xi, t) \mathrm{d} \xi \\
& -\frac{\pi^{2}}{4} \int_{0}^{1} \bar{u}^{T}(\xi, t)\left(D^{T} P+P D\right) \bar{u}(\xi, t) \mathrm{d} \xi
\end{aligned}
$$


Combining (49) and (53), and the fact that $\dot{V}_{3}=0$, we obtain

$$
\dot{V}(t)-\alpha V(t) \leq \int_{0}^{1}\left[\begin{array}{c}
X^{T}(t) \\
u^{T}(\xi, t) \\
\bar{u}^{T}(\xi, t) \\
d
\end{array}\right]^{T}\left[\begin{array}{cccc}
\Xi_{1} & P D K_{1} & -P D K_{1} & P B \\
* & \Xi_{2} & -K_{2}^{T} D^{T} P & 0 \\
* & * & -\frac{\pi^{2}}{4}\left(D^{T} P+P D\right) & -P D \\
* & * & * & -\alpha Q
\end{array}\right]\left[\begin{array}{c}
X^{T}(t) \\
u^{T}(\xi, t) \\
\bar{u}^{T}(\xi, t) \\
d
\end{array}\right] \mathrm{d} \xi,
$$

where

$$
\begin{aligned}
& \Xi_{1}=P\left(A+B K_{1}\right)+\left(A+B K_{1}\right)^{T} P-\alpha P, \\
& \Xi_{2}=P\left(D K_{2}+G\right)+\left(D K_{2}+G\right)^{T} P-\alpha P .
\end{aligned}
$$

In view of (41) and (42), we get

$$
\dot{V}(t)-\alpha V(t)<0 .
$$

Multiplying both the left side and right side of inequality (56) by $e^{-\alpha t}$ and integrating (56) we have

$$
V(t)<e^{\alpha t} V(0) .
$$

Making $\widetilde{P}=R^{-(1 / 2)} P R^{-(1 / 2)}$, we obtain

$$
\begin{aligned}
& \lambda_{\min }(\widetilde{P})\left[x^{T}(t) R x(t)+\int_{0}^{1} u^{T}(\xi, t) R u(\xi, t) \mathrm{d} \xi\right] \\
& \leq x^{T}(t) P x(t)+\int_{0}^{1} u^{T}(\xi, t) P u(\xi, t) \mathrm{d} \xi+d^{T} Q d<e^{\alpha t} V(0), \\
& x^{T}(0) P x(0)+\int_{0}^{1} u^{T}(\xi, 0) P u(\xi, 0) \mathrm{d} \xi+d^{T} Q d \\
& \leq \lambda_{\max }(\widetilde{P})\left[x^{T}(0) R x(0)+\int_{0}^{1} u^{T}(\xi, 0) R u(\xi, 0) \mathrm{d} \xi\right] \\
& \quad+\lambda_{\max }(Q) d^{T} d \leq \lambda_{\max }(\widetilde{P})\left(x^{T}(0) R x(0)+\|u(\xi, 0)\|_{L_{2}, R}^{2}\right) \\
& \quad+\lambda_{\max }(Q) w .
\end{aligned}
$$

It is known that

$$
\begin{aligned}
& x^{T}(t) R x(t)+\|u(\xi, t)\|_{L_{2}, R}^{2} \\
& <\frac{e^{\alpha t}\left[\lambda_{\max }(\widetilde{P})\left(x^{T}(0) R x(0)+\|u(\xi, 0)\|_{L_{2}, R}^{2}\right)+\lambda_{\max }(Q) w\right]}{\lambda_{\min }(\widetilde{P})} .
\end{aligned}
$$

From condition (43), we get the conclusion

$$
x^{T}(t) R x(t)+\|u(\xi, t)\|_{L_{2}, R}^{2}<c_{2}^{2} .
$$

The proof is completed.

Corollary 2. The finite-time boundedness problem from Theorem 2 is solvable if there exists a positive definite

symmetric matrix $S$, the matrices $L_{1}$ and $L_{2}$, and positive scalars $\lambda_{1}$ and $\lambda_{2}$. By fixing a nonnegative scalar $\alpha$, the following LMIs satisfy:

$$
\begin{aligned}
& {\left[\begin{array}{cccc}
\Phi_{1} & D L_{1} & -D L_{1} & B S \\
* & \Phi_{2} & -L_{2}^{T} D^{T} & 0 \\
* & * & -\frac{\pi^{2}}{4}\left(S D^{T}+D S\right) & -D S \\
& & & \\
* & * & * & -\alpha Q
\end{array}\right]<0,} \\
& S D^{T}+D S>0, \\
& S-R^{-1}<0, \\
& {\left[\begin{array}{cc}
-S & R^{-(1 / 2)} \\
* & -\lambda_{1} I
\end{array}\right]<0,} \\
& Q<\lambda_{2} I, \\
& \lambda_{1} c_{1}^{2}+\lambda_{2} w<c_{2}^{2} e^{-\alpha T},
\end{aligned}
$$

where

$$
\begin{aligned}
& \Phi_{1}=A S+S A^{T}+B L_{1}+L_{1}^{T} B^{T}-\alpha S, \\
& \Phi_{2}=G S+S G^{T}+D L_{2}+L_{2}^{T} D^{T}-\alpha S .
\end{aligned}
$$

Proof. Let $S=P^{-1}$, pre and postmultiply (41) by S. Set $L_{1}=$ $K_{1} S$ and $L_{2}=K_{2} S$. Condition (61) is equivalent to that (41) holds. Condition (62) can be transformed to (42) with $S=P^{-1}$.

Making $\widetilde{P}=R^{-(1 / 2)} P R^{-(1 / 2)}$ and by imposing the conditions

$$
\begin{aligned}
I & <\widetilde{P}<\lambda_{1} I, \\
Q & <\lambda_{2} I,
\end{aligned}
$$

where $\lambda_{1}$ and $\lambda_{2}$ are positive scalars, we have 


$$
\lambda_{1}^{-1} R^{-1}<S<R^{-1}
$$

which is equivalent to (63) and (64) hold, and condition (43) is equivalent to

$$
\lambda_{1} c_{1}^{2}+\lambda_{2} w<c_{2}^{2} e^{-\alpha T}
$$

In this case, the matrix $P=S^{-1}$, and the boundary feedback controller gains are $K_{1}=L_{1} S^{-1}$ and $K_{2}=L_{2} S^{-1}$.

The proof is completed.

\section{Results of Simulation}

The relevant parameters of the coupled parabolic PDE-ODE system (1) are listed below:

$$
\left\{\begin{array}{l}
x(t)=\left[\begin{array}{cc}
1 & 0 \\
-0.5 & 0.2
\end{array}\right] x(t)+\left[\begin{array}{cc}
0.8 & 0.5 \\
-0.4 & 0.8
\end{array}\right] u_{\xi}(0, t), \\
u_{t}(\xi, t)=\left[\begin{array}{cc}
0.1 & 0 \\
0 & 0.1
\end{array}\right] u_{\xi \xi}(\xi, t)+\left[\begin{array}{cc}
1 & 0.5 \\
-0.5 & 1.2
\end{array}\right] u(\xi, t),
\end{array}\right.
$$

where

$$
\begin{gathered}
x(t)=\left[\begin{array}{l}
x_{1}(t) \\
x_{2}(t)
\end{array}\right], \\
u(\xi, t)=\left[\begin{array}{l}
u_{1}(\xi, t) \\
u_{2}(\xi, t)
\end{array}\right] .
\end{gathered}
$$

The initial values satisfy

$$
\begin{aligned}
x(0) & =\left[\begin{array}{c}
2.2 \\
2
\end{array}\right], \\
u(\xi, 0) & =\left[\begin{array}{c}
0.5 \cos (\pi \xi)+0.5 \sin (\pi \xi) \\
0.5 \sin (\pi \xi)
\end{array}\right],
\end{aligned}
$$

and the boundary control conditions are

$$
\begin{aligned}
u(0, t) & =0, \\
u_{\xi}(1, t) & =U(t)+d(t) .
\end{aligned}
$$

Example 1. Finite-time boundedness of the coupled parabolic PDE-ODE systems with time-varying boundary disturbances.

For given $c_{1}=3.2, c_{2}=23, T=1, R=I, w=1$, and $d(t)=[\cos (5 t), \sin (5 t)]^{T}$. Fixing $\alpha=2.5$, we get feasible solutions from the corresponding linear matrix inequalities in Corollary 1.

We obtain that $\lambda_{1}=4.0499, \beta=0.8174$, and $\gamma=0.9475$, and the corresponding matrices are

$$
\begin{aligned}
P & =\left[\begin{array}{cc}
1.0096 & -0.0071 \\
* & 1.0093
\end{array}\right], \\
K_{1} & =\left[\begin{array}{cc}
-3.2881 & 0.0080 \\
-0.0115 & -3.2810
\end{array}\right], \\
K_{2} & =\left[\begin{array}{ll}
-1.7137 & -0.0193 \\
-0.0150 & -1.2776
\end{array}\right] .
\end{aligned}
$$

The designed Neumann boundary feedback controller $U(t)$ is given as

$$
\begin{aligned}
U(t)= & {\left[\begin{array}{cc}
-3.2881 & 0.0080 \\
-0.0115 & -3.2810
\end{array}\right] x(t) } \\
& +\int_{0}^{1}\left[\begin{array}{rr}
-1.7137 & -0.0193 \\
-0.0150 & -1.2776
\end{array}\right] u(\xi, t) \mathrm{d} \xi .
\end{aligned}
$$

As a result, system (1) in the presence of time-varying boundary disturbance is finite-time-bounded with respect to $c_{1}=3.2, c_{2}=23, T=1, R=I$, and $w=1$ for the existence of the Neumann boundary feedback controller (76). To illustrate the availability of the designed boundary controller, the open-loop responses of $x(t)$ and $u(\xi, t)$ with time-varying boundary disturbance $d(t)$ are shown in Figure 2, and the trajectories of $x(t)$ and $u(\xi, t)$ along with the Neumann boundary feedback controller (76) are then shown in Figure 3 .

Example 2. Finite-time boundedness of the coupled parabolic PDE-ODE systems with time-invariant boundary disturbances.

In order to illustrate that the proposed method in Theorem 2 is less conservative than Theorem 1 , the values $c_{1}$, $c_{2}, R, T$, and $w$ are selected as the same as in Example 1. Fixing $\alpha=2.5$, we get feasible solutions from the corresponding linear matrix inequalities in Corollary 2.

We found that $\lambda_{1}=4.1624$ and $\lambda_{2}=0.6768$, and the corresponding matrices

$$
\begin{aligned}
P & =\left[\begin{array}{cc}
1.0196 & 0.0002 \\
* & 1.0200
\end{array}\right], \\
Q & =\left[\begin{array}{cc}
0.4038 & 0.0084 \\
* & 0.4056
\end{array}\right], \\
K_{1} & =\left[\begin{array}{cc}
-1.6008 & -0.3182 \\
0.0714 & -1.5605
\end{array}\right], \\
K_{2} & =\left[\begin{array}{cc}
-0.9068 & -0.0184 \\
-0.0216 & -1.0824
\end{array}\right] .
\end{aligned}
$$

The designed Neumann boundary feedback controller $U(t)$ is given by

$$
\begin{aligned}
U(t)= & {\left[\begin{array}{rr}
-1.6008 & -0.3182 \\
0.0714 & -1.5605
\end{array}\right] x(t) } \\
& +\int_{0}^{1}\left[\begin{array}{rr}
-0.9068 & -0.0184 \\
-0.0216 & -1.0824
\end{array}\right] u(\xi, t) \mathrm{d} \xi .
\end{aligned}
$$

Therefore, system (1) in the presence of time-invariant boundary disturbance is finite-time-bounded with respect to $\left(c_{1}=3.2, c_{2}=23, T=1, R=I\right.$, and $\left.w=1\right)$ under the Neumann boundary feedback controller (78). The trajectories of $x(t)$ and $u(\xi, t)$ along with the Neumann boundary feedback controller (78) are then shown in Figure 4.

The simulation results of the two examples show that the smaller controller gains $K_{1}$ and $K_{2}$ are obtained in Example 2 to ensure the finite-time boundedness of system (1), which 


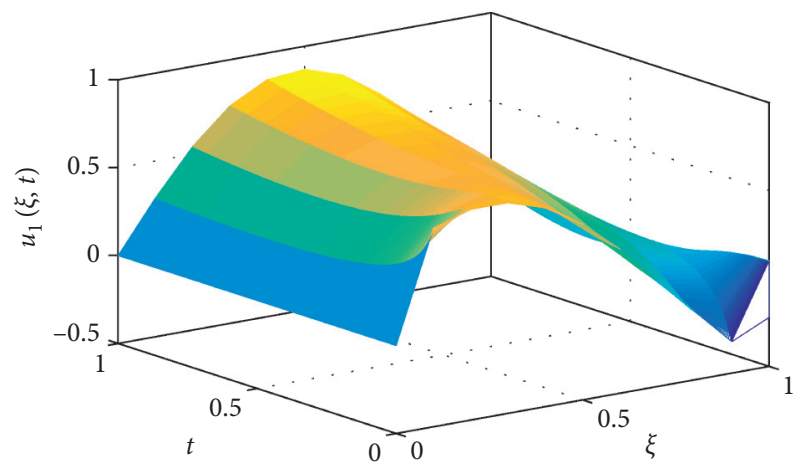

(a)

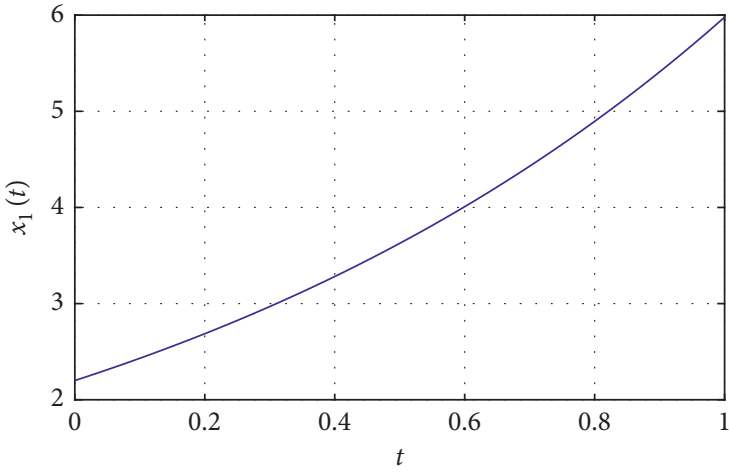

(c)

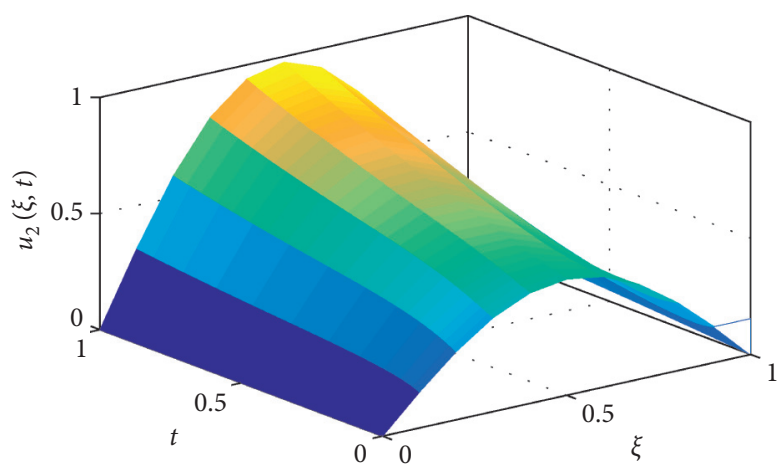

(b)

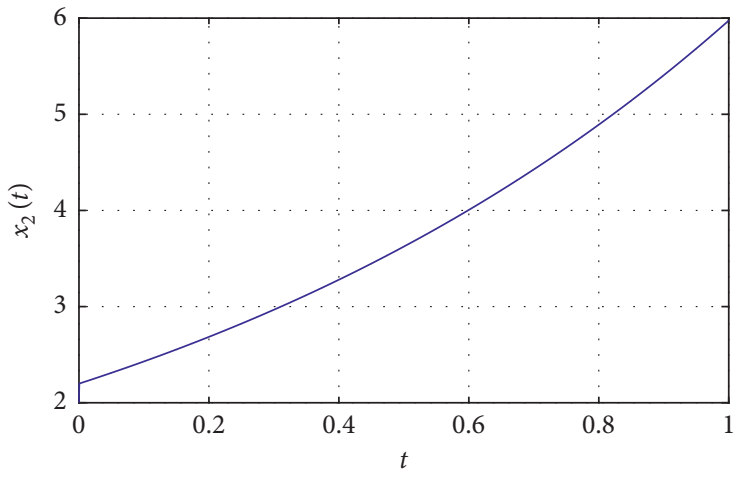

(d)

Figure 2: Open-loop responses of $x(t)$ and $u(\xi, t)$ with time-varying boundary disturbance.

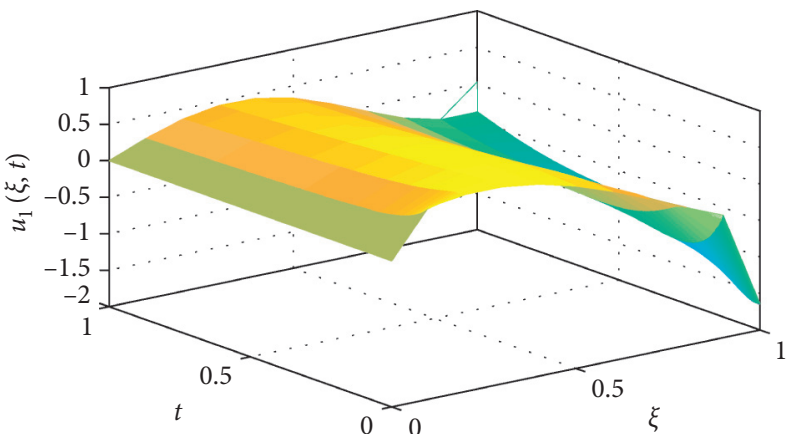

(a)

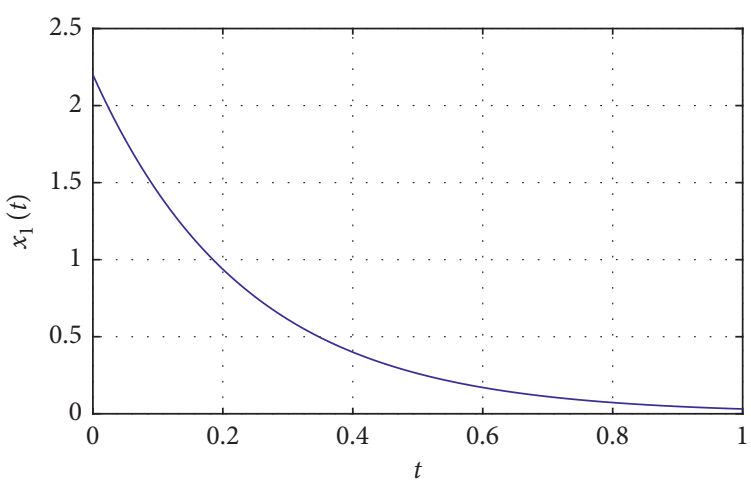

(c)

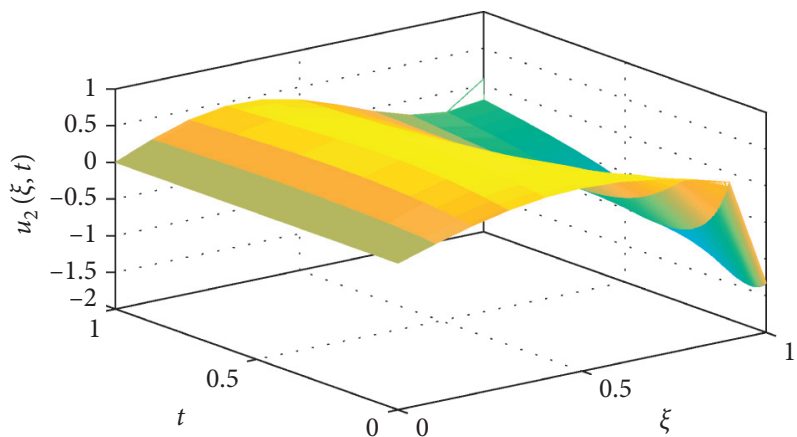

(b)

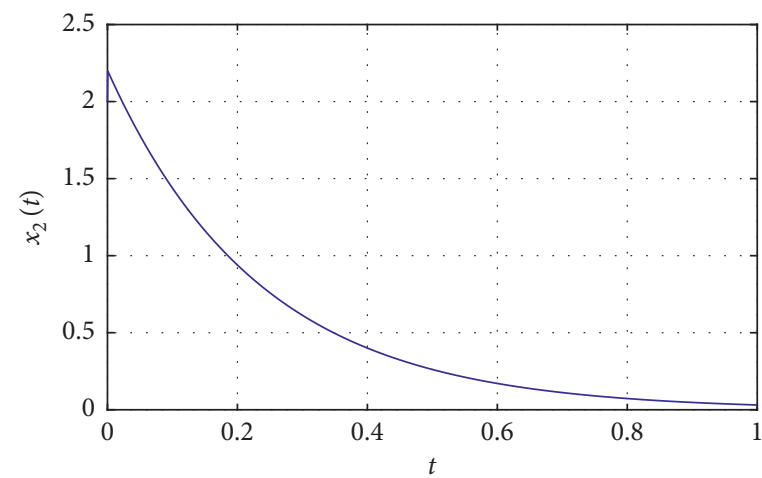

(d)

FIgURE 3: Closed-loop responses of $x(t)$ and $u(\xi, t)$ with time-varying boundary disturbance. 


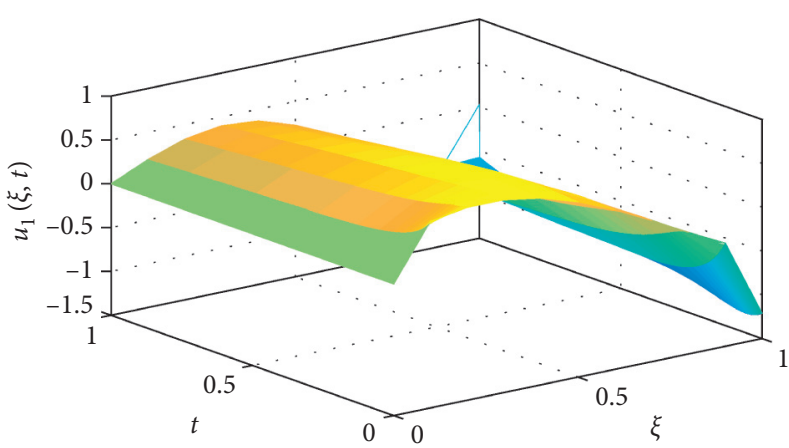

(a)

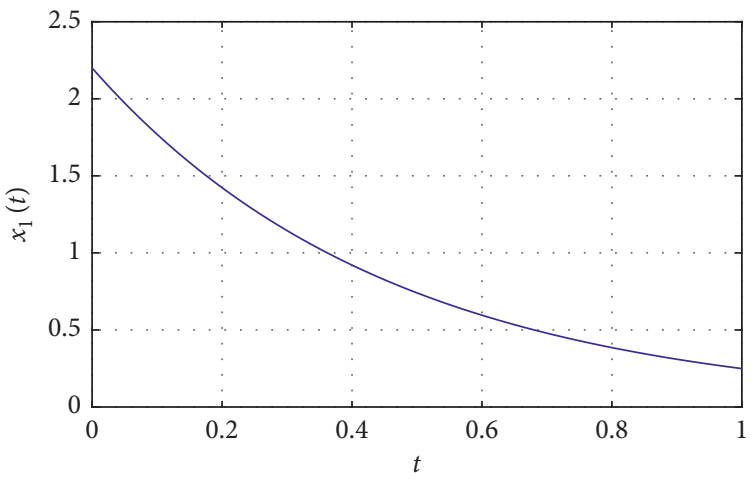

(c)

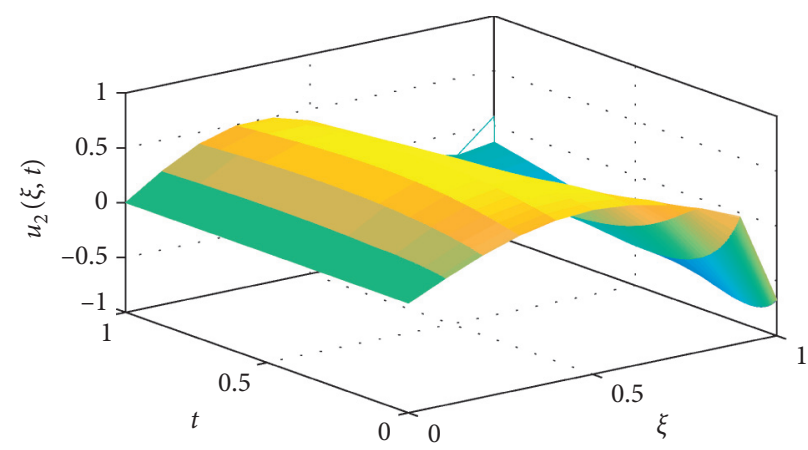

(b)

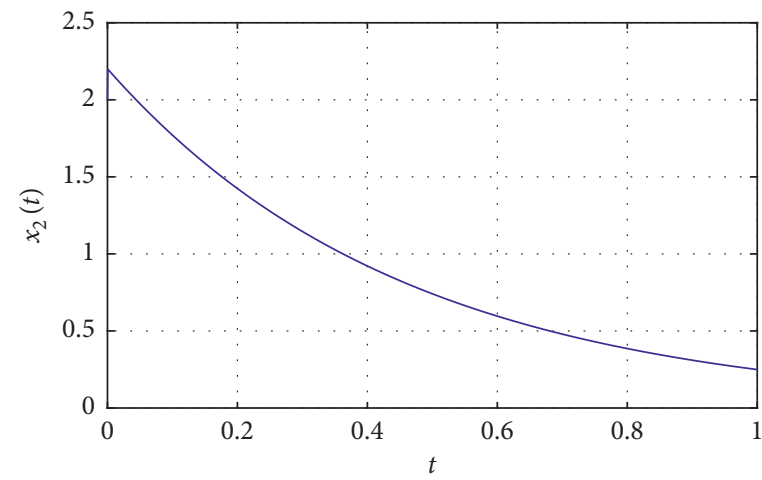

(d)

Figure 4: Closed-loop responses of $x(t)$ and $u(\xi, t)$ with time-invariant boundary disturbance.

imply that Theorem 2 can get a less conservative result than Theorem 1 in the presence of time-invariant boundary disturbances.

\section{Summary}

The finite-time-bounded control problem for coupled parabolic PDE-ODE systems with external disturbances at the Neumann boundary control end is discussed in this paper. First, a Neumann boundary feedback controller is designed, and by using Lyapunov-like function method and scaling technique of inequalities, we then show how to obtain sufficient conditions for finite-time boundedness of the closed-loop parabolic PDE-ODE couples with time-varying boundary disturbances and time-invariant boundary disturbances, respectively. The proposed design conditions are turned to feasibility problems of linear matrix inequalities (LMIs) and we end up with some numerical simulations validating the results.

\section{Data Availability}

The data used to support the findings of this study are included within the article.

\section{Conflicts of Interest}

The authors declare that there are no conflicts of interest regarding the publication of this paper.

\section{Acknowledgments}

This work was supported by the National Natural Science Foundation of China (NSFC) under Grant nos. 61633019 and 61473252 .

\section{References}

[1] I. Lasiecka, Mathematical Control Theory of Coupled PDEs, SIAM, Philadelphia, PA, USA, 2002.

[2] R. Lifshitz and M. L. Roukes, "Thermoelastic damping in micro-and nanomechanical systems," Physical Review B, vol. 61 , no. 8, pp. 5600-5609, 2000.

[3] M. Krstic and A. Smyshlyaev, Boundary Control of PDEs: A Course on Backstepping Designs, SIAM, San Diego, CA, USA, 2008.

[4] M. Krstic and A. Smyshlyaev, "Backstepping boundary control for first-order hyperbolic PDEs and application to systems with actuator and sensor delays," Systems \& Control Letters, vol. 57, no. 9, pp. 750-758, 2008.

[5] G. A. Susto and M. Krstic, "Control of PDE-ODE cascades with Neumann interconnections," Journal of the Franklin Institute, vol. 347, no. 1, pp. 284-314, 2010.

[6] M. Krstic, "Control of an unstable reaction-diffusion PDE with long input delay," Systems and Control Letters, vol. 58, no. 10-11, pp. 773-782, 2009.

[7] Z. Zhou and C. Xu, "Stabilization of a second order ODE-heat system coupling at intermediate point," Automatica, vol. 60, pp. 57-64, 2015.

[8] Y. P. Guo and J. J. Liu, "Stabilization of ODE-Schrodinger cascaded systems subject to boundary control matched 
disturbance," Electronic Journal of Differential Equations, vol. 248, pp. 1-22, 2015.

[9] J.-M. Wang, J.-J. Liu, B. Ren, and J. Chen, "Sliding mode control to stabilization of cascaded heat PDE-ODE systems subject to boundary control matched disturbance," Automatica, vol. 52, pp. 23-34, 2015.

[10] P. Dorato, "Short-time stability in linear time-varying systems," Proceedings of the IRE International Convention Record Part, vol. 4, pp. 83-87, 1961.

[11] L. Weiss and E. F. Infante, "On the stability of systems defined over a finite time interval," Proceeding of the National Academy of Sciences of the United States of America, vol. 54, no. 1, pp. 44-48, 1965.

[12] F. S. Filippo and P. Dorato, "Short-time parameter optimization with flight control applications," Automatica, vol. 10, no. 5, pp. 425-430, 1974.

[13] F. Amato, M. Ariola, and C. Cosentino, "Finite-time stability of linear time-varying systems: analysis and controller design," IEEE Transactions on Automatic Control, vol. 55, no. 4, pp. 1003-1008, 2010.

[14] F. Amato and M. Ariola, "Finite-time control of discrete-time linear systems," IEEE Transactions on Automatic Control, vol. 50, no. 5, pp. 724-729, 2005.

[15] Y. Guo, Y. Yao, S. Wang, K. Ma, K. Liu, and J. Guo, "Inputoutput finite-time stabilization of linear systems with finitetime boundedness," ISA Transactions, vol. 53, no. 4, pp. 977-982, 2014.

[16] F. Amato, M. Ariola, and F. Ambrosino, "Finite-time stability of linear systems: an approach based on polyhedral Lyapunov functions," IET Control Theory \& Applications, vol. 4, no. 9, pp. 1767-1774, 2010.

[17] W. P. Xue and W. J. Mao, “Admissible finite-time stability and stabilization of uncertain discrete singular systems," Journal of Dynamic Systems, Measurement, and Control, vol. 135, no. 3, pp. 0310181-0310186, 2013.

[18] S. P. Bhat and D. S. Bernstein, "Finite-time stability of continuous autonomous systems," SIAM Journal on Control and Optimization, vol. 38, no. 3, pp. 751-766, 2000.

[19] E. Moulay and W. Perruquetti, "Finite time stability and stabilization of a class of continuous systems," Journal of Mathematical Analysis and Applications, vol. 323, no. 2, pp. 1430-1443, 2006.

[20] W. Chen and L. C. Jiao, "Finite-time stability theorem of stochastic nonlinear systems," Automatica, vol. 46, no. 12, pp. 2105-2108, 2010.

[21] E. P. Ryan, "Finite-time stabilization of uncertain nonlinear planar systems," Dynamics and Control, vol. 1, no. 1, pp. 83-94, 1991.

[22] X. Li, D. W. C. Ho, and J. Cao, "Finite-time stability and settling-time estimation of nonlinear impulsive systems," Automatica, vol. 99, pp. 361-368, 2019.

[23] X. Yang, X. Li, and J. Cao, "Robust finite-time stability of singular nonlinear systems with interval time-varying delay," Journal of the Franklin Institute, vol. 355, no. 3, pp. 1241-1258, 2018.

[24] G. Bartolini, A. Ferrara, and V. I. Utkin, "Adaptive sliding mode control in discrete-time systems," Automatica, vol. 31, no. 5, pp. 769-773, 1995.

[25] M. R. James, J. S. Baras, and R. J. Elliott, "Risk-sensitive control and dynamic games for partially observed discretetime nonlinear systems," IEEE Transactions on Automatic Control, vol. 39, no. 4, pp. 780-792, 1994.

[26] J.-M. Coron, "On the stabilization in finite time of locally controllable systems by means of continuous time-varying feedback law," SIAM Journal on Control and Optimization, vol. 33, no. 3, pp. 804-833, 1995.

[27] X. Li and W. Mao, "Finite-time stability and stabilisation of distributed parameter systems," IET Control Theory \& Applications, vol. 11, no. 5, pp. 640-646, 2017.

[28] M. Li and W. Mao, "DC-boundary finite-time stabilisation for distributed parameter systems," IET Control Theory \& Applications, vol. 14, no. 15, pp. 2186-2196, 2020.

[29] F. Amato, M. Ariola, and P. Dorato, "Finite-time control of linear systems subject to parametric uncertainties and disturbances," Automatica, vol. 37, no. 9, pp. 1459-1463, 2001.

[30] F. Amato, M. Ariola, and C. Cosentino, "Finite-time control of linear time-varying systems via output feedback," in Proceedings of the 2005 American Control Conference, pp. 4722-4726, Portland, OR, USA, June 2005.

[31] Y. G. Sun and J. Xu, "Finite-time boundedness and stabilization of networked control systems with time delay," Mathematical Problems in Engineering, vol. 2012, Article ID 705828, , 2012.

[32] H. B. Du, X. Z. Lin, and S. H. Li, "Finite-time boundedness and stabilization of switched linear systems," Kybernetika, vol. 46, no. 5, pp. 870-889, 2010.

[33] J. LaSalle and S. Lefschetz, Stability by Liapunov's Direct Method with Applications, Academic Press, New York, NY, USA, 1961.

[34] G. H. Hardy, J. E. Littlewood, and G. Polya, Inequalities, Cambridge University Press, Cambridge, UK, 2nd edition, 1952. 